\begin{tabular}{|c|l|}
\hline Title & Size Dependent Carrier Dynamics in CdS Nanoparticles by Femtosecond V isible Pump/R-Probe Measurements \\
\hline Author(s) & Y agi, Ichizo; Mikami, Kensuke; Ebina, Kojiro; Okamura, Masayuki; U osaki, Kohei \\
\hline Citation & $\begin{array}{l}\text { Journal of Physical Chemistry B, 110(29), 14192-14197 } \\
\text { https://doi.org/10.1021/j061073t }\end{array}$ \\
\hline Issue Date & 2006-07-27 \\
\hline Doc URL & http://hdl.handle.net/2115/50222 \\
\hline Type & article \\
\hline File Information & JPCB110-29_14192-14197.pdf \\
\hline
\end{tabular}

Instructions for use 


\title{
Size-Dependent Carrier Dynamics in CdS Nanoparticles by Femtosecond Visible-Pump/ IR-Probe Measurements
}

\author{
Ichizo Yagi, ${ }^{\dagger, \hbar, \S}$ Kensuke Mikami, ${ }^{\dagger}$ Kojiro Ebina, ${ }^{\dagger}$ Masayuki Okamura, ${ }^{\dagger}$ and Kohei Uosaki $*, \dagger$ \\ Physical Chemistry Laboratory, Division of Chemistry, Graduate School of Science, Hokkaido University, \\ N10W8 Kita-ku, Sapporo 060-0810, Japan, PRESTO, Japan Science and Technology Agency (JST), \\ Kawaguchi 332-0012, Japan, and FC-Cubic, AIST, 2-41-6 Aomi, Koto-ku, Tokyo 135-0064, Japan
}

Received: February 20, 2006; In Final Form: April 26, 2006

\begin{abstract}
Ultrafast photoexcited carrier dynamics in CdS nanoparticles prepared by an AOT/n-heptane reversed micelle system were investigated by a femtosecond visible-pump/mid-IR probe technique. A mid-IR probe beam was found to mainly probe the ultrafast dynamics of photoexcited electrons in the conduction band. Dispersions of CdS nanoparticles with 8 different mean diameters from 2.9 to $4.1 \mathrm{~nm}$ were prepared by tuning the mole ratio between water and $\mathrm{AOT}\left(W=\left[\mathrm{H}_{2} \mathrm{O}\right] /[\mathrm{AOT}]\right)$ in the reversed micelle systems. The excited state lifetime strongly depended on the mean size of CdS nanoparticles with a maximum around a mean diameter of 3.5 $\mathrm{nm}$. This result was explained by considering the balance between the carrier recombination rates via surface states and those via interior states. The relationship between the excited state lifetime and the size of CdS nanoparticles was drastically changed when the surface was terminated by thiol molecules.
\end{abstract}

\section{Introduction}

Interest has been shown in quantum-confined materials over the past two decades because of their unique electronic and optical properties. Semiconductor quantum dots (QD) have attracted much attention because their band gap energy can be tuned by controlling their size and shape. Such tunability can be useful for a wide range of applications, including solar energy conversion, optical devices, and optical labels in biochemical systems. The static and dynamic properties of semiconductor QDs, especially II-VI semiconductor QDs, such as CdSe and $\mathrm{CdS}$ nanoparticles, have been investigated by various techniques. ${ }^{1}$ Transient absorption measurement has recently become an important tool for monitoring excited carrier dynamics in semiconductor nanoparticles. An understanding of excited carrier dynamics is important not only for device application but also for general photophysics of zero-dimensional systems.

Several groups have recently studied the relaxation dynamics of CdSe nanoparticles in terms of their surface properties and size dependence by femtosecond pump/probe spectroscopy. Guyot-Sionnest and co-workers ${ }^{2,3}$ demonstrated that the dynamic behavior of excited electrons could be monitored by using a mid-IR probe beam, in contrast to the complex relaxation behaviors in the visible probe measurements due to the contributions from both electrons and holes, as has been reported. ${ }^{4}$ They showed that the peak energy and bandwidth of transient mid-IR spectra for CdSe nanoparticles depended on their mean size and size distribution. Klimov et al. ${ }^{4-7}$ showed that intraband optical excitation could be used to separate the contributions of electron's and hole's transients by three-pulse pump-probe experiments. Burda et al. ${ }^{8,9}$ reported further experimental evidence that the transient mid-IR and near-IR behaviors could be correlated to electron and hole transients,

* Address correspondence to this author. Fax: +81-11-706-3440 E-mail: uosaki@PCL.sci.hokudai.ac.jp.

† Hokkaido University.

$\doteqdot$ PRESTO.

$\S$ FC-Cubic respectively. Recently, the difference between ultrafast spectroscopic behaviors of semiconductor nanodots and nanorods has been actively investigated. ${ }^{10-12}$ These investigations indicated that tunable near-IR and mid-IR probes can monitor the ultrafast dynamic behaviors of excited holes and electrons, respectively.

Photophysics and photochemistry of CdS nanoparticles have also been investigated in detail, because of the ease in their preparation and the availability of a wide variety of preparation methods. Exciton dynamics in CdS nanoparticles have been investigated mainly by time-resolved photoluminescence and transient absorption. ${ }^{13}$ Although Zhang et al. ${ }^{14,15}$ have investigated in detail the charge carrier dynamics of $\mathrm{CdS}$ nanoparticles dependent on various parameters, such as size, excitation intensity, and surface termination of CdS nanoparticles, using visible-pump visible-probe measurement, the transient behavior monitored by visible probe does not provide information of independent contributions from holes and electrons, as mentioned above. Thus, it is important to investigate the carrier dynamics in CdS nanoparticles by using probe beams in the mid-IR to near-IR wavelength region.

In the present study, visible-pump/mid-IR-probe transient absorption measurements were carried out for dispersions of $\mathrm{CdS}$ nanoparticles of 8 different carefully controlled mean sizes, which were prepared by the reversed micelle method. The origin of mid-IR absorption was clearly assigned by measuring the decay behaviors in solutions containing electron or hole acceptors. A volcano-type relationship between relaxation lifetime and mean size of CdS nanoparticles was obtained. The effect of surface termination on the electron dynamics was also investigated.

\section{Experimental Section}

I. Materials. Ethanol (superpure grade), 1-butanol (superpure grade), acetone (superpure grade), bis(2-ethylhexyl) sodium sulfosuccinate (AOT, pure grade), triethanolamine (superpure 


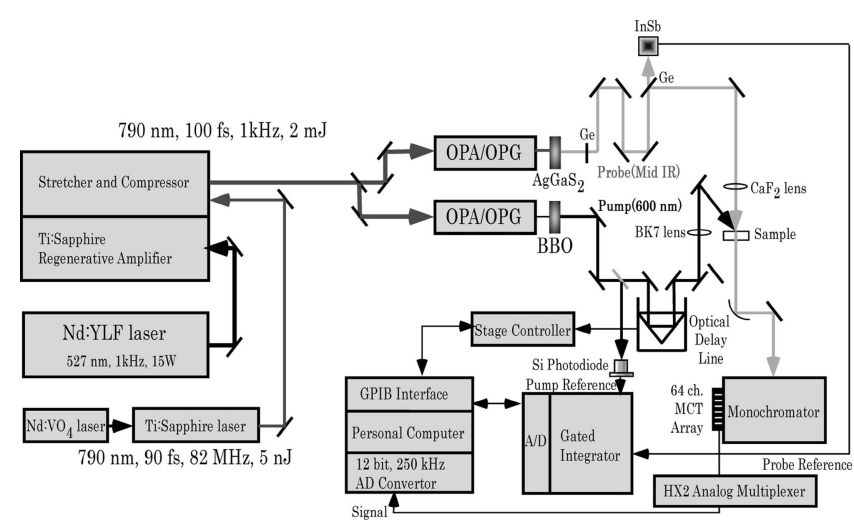

Figure 1. Block diagram of the femtosecond visible-pump/mid-IRprobe spectroscopic system.

grade), and $\mathrm{Na}_{2} \mathrm{~S} \cdot 9 \mathrm{H}_{2} \mathrm{O}$ (superpure grade) were purchased from Wako Pure Chemicals. $\mathrm{Cd}\left(\mathrm{ClO}_{4}\right)_{2} \cdot 6 \mathrm{H}_{2} \mathrm{O}$ was obtained from Kishida Chemicals. 2-Mercaptoethanesulfonate (97\%) was obtained from Aldrich, and toluene (spectroscopy grade), $n$-heptane (spectroscopy grade), and methanol (spectroscopy grade) were purchased from Dojindo Laboratory. 1,4-Benzoquinone, 1,4,-aminothiophenol, thiophenol, and 1,1'-dimethyl4,4'-bipyridinium dichlodide (methyl viologen, $>95 \%$ ) were purchased from TCI. All chemicals were used without further purification. Ultrapure water was obtained by using a Milli-Q water purification system (Millipore). Ar (99.999\%) and $\mathrm{N}_{2}$ (99.99\%) were obtained from air-water.

II. Preparation of CdS Nanoparticles. CdS nanoparticles were prepared in AOT/ $n$-heptane reversed micelles. ${ }^{16}$ Typically, $100 \mathrm{~mL}$ of $n$-heptane solution of $0.2 \mathrm{M}$ AOT was prepared in two separate Schlenck tubes. An aqueous solution of $\mathrm{Cd}\left(\mathrm{ClO}_{4}\right)_{2}$. $6 \mathrm{H}_{2} \mathrm{O}(0.4 \mathrm{M})$ was added to one solution, while an aqueous solution of $\mathrm{Na}_{2} \mathrm{~S} \cdot 9 \mathrm{H}_{2} \mathrm{O}(0.3 \mathrm{M})$ was added to the other solution, to give a molar ratio of $W=\left[\mathrm{H}_{2} \mathrm{O}\right] /[\mathrm{AOT}]$ for both solutions. After each solution had been stirred for $1-2 \mathrm{~h}$, the solutions were mixed together and stirred for another $1 \mathrm{~h}$, resulting in the formation of CdS nanoparticles in the reversed micelles. The dispersions of as-prepared CdS nanoparticles were used as samples.

Thiol-capped CdS nanoparticles were prepared as follows. ${ }^{17}$ The terminal agent 2-mercaptoethanesulfonate was added to a $\mathrm{CdS} / \mathrm{AOT} / n$-heptane solution, and the solution was stirred for $12 \mathrm{~h}$. Pyridine was then added to destroy the AOT reversed micelles, resulting in the formation of precipitate, i.e., thiolcapped CdS nanoparticles. After removal of the top clear layer of the solution by decantation, the thiol-capped CdS nanoparticles were washed successively with pyridine, $n$-heptane, diethyl ether, 1-butanol, acetone, and methanol. Thiol-terminated CdS nanoparticles were dispersed in deuterium oxide $\left(\mathrm{D}_{2} \mathrm{O}\right)$.

UV-visible spectra of nanoparticles were obtained by a Hitachi U-3300 spectrometer. FT-IR spectra were obtained by using Biorad FTS-30, and the optimal IR wavelength for the time-resolved IR probe measurement was determined to avoid strong IR absorption of AOT, water, and $n$-heptane.

III. Time-Resolved Visible-Pump/IR-Probe Measurements. Observations of picosecond transitional absorption change were performed with a femtosecond time-resolved visible-pump/IR-probe system ${ }^{18,19}$ as schematically shown in Figure 1. In all measurements, excitation wavelength was 395 $\mathrm{nm}(3.13 \mathrm{eV})$ and probe wavelength was tuned to avoid large absorption of $\mathrm{H}_{2} \mathrm{O}$ and $\mathrm{D}_{2} \mathrm{O}$. The pulse train of $120 \mathrm{fs}$ pulses of $2 \mathrm{~mJ}$ energy with a $1 \mathrm{kHz}$ repetition rate was generated by a
Ti:sapphire regenerative multipath amplifier system (Quantronics, model 4812RGA/4823S/C), which was synchronously pumped by a mode-locked YLF laser (Quantronics, 527DP$\mathrm{H}$ ). The seed pulses (400 $\mu \mathrm{J}$ energy, $100 \mathrm{fs}$ pulses centered at $790 \mathrm{~nm}$ with a repetition frequency of $82 \mathrm{MHz}$ ) were supplied by a mode-locked Ti:sapphire laser (Spectra-Physics, Tsunami: 3960-L2S) pumped by an $\mathrm{Nd}: \mathrm{YVO}_{4}$ laser (SpectraPhysics, Millenia-Vs). The final output of the laser system was split into two equivalent beams to generate the pump and probe beams for the TRIR measurements. The probe beam $(2.5-6.0$ $\mu \mathrm{m}$, bandwidth $200 \mathrm{~cm}^{-1}, 0.1-0.8 \mu \mathrm{J}$ pulse $^{-1}$ ) was obtained by difference frequency generation in an $\mathrm{AgGaSe}_{2}$ crystal (thickness $1 \mathrm{~mm}$ ) of signal and idler beams from an optical parametric amplification/optical parametric generation (OPA/ OPG) system (Quantronix, TOPAS 8034) pumped by one beam. The other beam was used to generate a pump beam $(395 \mathrm{~nm}$, $0.5-9.0 \mu \mathrm{J}$ puls $^{-1}$ ) by second harmonic generation in a BBO crystal (thickness $1 \mathrm{~mm}$ ). The wavelengths of the pump and probe beams represent the central wavelengths of both beams since the bandwidths of femtosecond pulses are relatively broad (fwhm $\sim 200 \mathrm{~cm}^{-1}$ ). The probe beam was split into two beams. One beam of smaller intensity was directed into a reference channel with an InSb detector (Hamamatsu, P5172-200), and the other beam was passed through the sample and the IR-pass visible-blocking glass filters and then introduced to a monochromator (Bunko Keiki, M-25), which was coupled with a liquid nitrogen-cooled 64 element MCT linear array system (Infrared Associates, IR-6416). The pump and probe beams were noncollinearly focused with use of focal lenses and overlapped with each other in the sample cell with incident angles of $25^{\circ}$ and $0^{\circ}$ (surface normal), respectively. In all experiments, the sample solution was flowed in the thin layer cell at a flow rate of $2 \mathrm{~mL} \mathrm{~min}^{-1}$. The optical path length was limited to $1.0 \mathrm{~mm}$ by a Teflon spacer and $\mathrm{CaF}_{2}$ windows. The diameters of the pump and probe beams were 300 and $220 \mu \mathrm{m}$, respectively. The amplified outputs from the MCT array and the InSb detector were fed into a personal computer (NEC) via I/O interface and via a combination of a gated boxcar integrator (Stanford Research, SR-250) and a digitized with 12-bit A/D converter unit (Stanford Research, SR-245), respectively. Transmittance data were accumulated and recorded by a personal computer as normalized absorption change, $\Delta \mathrm{Abs}=\left(I_{0}-I\right) / I_{0}$, where $I_{0}$ and $I$ represent intensities of transmitted IR beam with and without visible excitation, respectively. Both $I$ and $I_{0}$ were normalized by the reference IR beam intensity within a given fluctuation, which was typically $\pm 5 \%$. The delay time between the pump and probe pulse was controlled by an optical delay line consisting of a retroreflector on a 1-axis translation stage (Sigma Koki) with $1 \mu \mathrm{m}$ (3.3 fs) resolution and $1 \mathrm{~ns}$ maximum delay.

\section{Results and Discussion}

I. Steady-State Absorption Spectra. Figure 2 shows UVvisible absorption spectra of the as-prepared $\mathrm{CdS}$ nanocluster dispersions in $n$-heptane with various values of $W$. As has already been reported, the absorption edge shifted to a shorter wavelength with a decrease in the value of $W$, indicating a change in the size of CdS nanoparticles. ${ }^{16,20}$ The mean diameter of the CdS nanoparticles for each sample was calculated from the exciton peak, which is defined as the position of the absorption maximum or shoulder, using tight-binding approximations with the assumption that the energy of the exciton peak in Figure 2 gives the band-gap energy. The calculated mean diameters of several CdS nanoparticle dispersions are sum- 


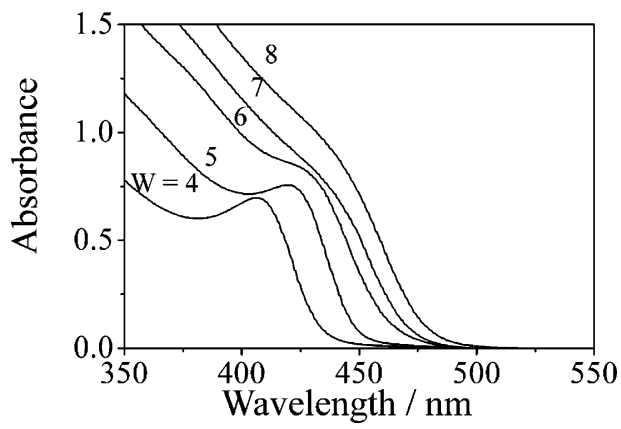

Figure 2. (a) UV-visible absorption spectra of CdS nanoparticle dispersions prepared by AOT $/ n$-hepane reversed micelle systems with various values of $\mathrm{W}\left(=[\mathrm{AOT}] /\left[\mathrm{H}_{2} \mathrm{O}\right]\right)$. (b) $\mathrm{UV}-$ visible absorption spectra of as-prepared (gray line) and thiol-capped (black line) CdS nanoparticles $(W=8)$.

TABLE 1: Correlation between the Calculated Mean Diameter of CdS Nanoparticles and the Value of $W=$ $\left[\mathrm{H}_{2} \mathrm{O}\right] /[\mathrm{AOT}]$

\begin{tabular}{ccc}
\hline$W$ & $\begin{array}{c}\text { exiton } \\
\text { peak/cm }\end{array}$ & $\begin{array}{c}\text { calcd } \\
\text { diameter/nm }\end{array}$ \\
\hline 4.0 & 406 & 2.9 \\
5.0 & 418 & 3.2 \\
6.0 & 426 & 3.4 \\
7.0 & 438 & 3.8 \\
8.0 & 446 & 4.1
\end{tabular}

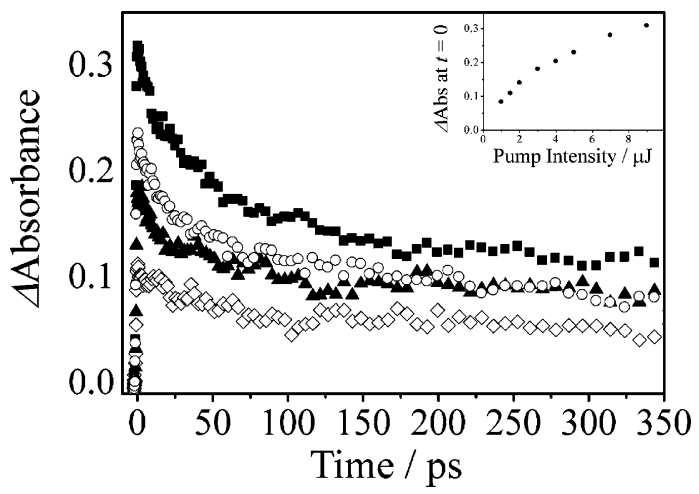

Figure 3. Excitation intensity-dependent photoinduced $\Delta \mathrm{Abs}$ relaxation dynamics of CdS nanoparticles prepared with the parameter of $W=8$ probed at $4.0 \mu \mathrm{m}$, following excitation at $395 \mathrm{~nm}$. Time-resolved IR transients correspond to the visible excitation intensities of 1.5 (open diamonds), 3.0 (solid triangles), 5.0 (open circles), and 9.0 $\mu \mathrm{J}$ (solid squares), respectively. The insets shows the correlation between the value of $\Delta \mathrm{Abs}$ at $t=0$ and the excitation intensity.

marized in Table 1. A broad absorption peak at around 3500 $\mathrm{cm}^{-1}$ due to the $\mathrm{O}-\mathrm{H}$ stretching vibration of water was observed in FT-IR spectra of the reversed micelle dispersions containing (a) $\mathrm{Cd}^{2+}$ and (b) $\mathrm{S}^{2-}$ and of the as-prepared CdS nanocluster $(W=8)$ dispersions in $n$-heptane. The intensity and shape of the $\mathrm{O}-\mathrm{H}$ stretching peak of the three spectra were identical, showing water was still prsent in the reversed micelle even after the formation of CdS nanoparticles most probably between $\mathrm{CdS}$ and AOT (see the Supporting Information).

II. Effect of Excitation Intensity. Figure 3 shows the IR absorption decay behaviors at various excitation intensities for the as-prepared $\mathrm{CdS}$ nanoparticle $(W=8)$ dispersion. The wavelength of the IR probe was set to $4 \mu \mathrm{m}(0.31 \mathrm{eV})$ to avoid large absorption by water around the CdS nanoparticles. $\Delta \mathrm{Abs}$ increased drastically at $t=0$ and decreased with biexponential decay in the following several hundred picoseconds at all excitation intensities. The inset in Figure 3 shows the excitation intensity dependence of $\Delta \mathrm{Abs}$ at $t=0$. The relaxation lifetimes of the fast and slow components were determined with biexponential decay fitting to be $30 \mathrm{ps}$ and $1.0 \mathrm{~ns}$, respectively, and the contribution of the fast component became larger with an increase in excitation intensity. Such excitation intensity dependencies have been reported for $\mathrm{CdS}$ and $\mathrm{CdSe}$ nanoparticles with both visible-pump/visible-probe and visible-pump/IR-probe transient absorption measurements. ${ }^{5,14,21,22}$ In the dispersions of $\mathrm{CdS}$ nanoparticles of smaller mean diameters, a linear correlation between $\Delta \mathrm{Abs}$ at $t=0$ and excitation intensity was also observed, but the threshold of the excitation intensity where the nonlinear correlation appears shifts toward larger excitation intensities than those for dispersions of $\mathrm{CdS}$ nanoparticles of larger mean diameters. Such nonlinearity appears to be due to the increase in the excitation photon density per CdS nanoparticle. In the present study, since we used a CdS nanoparticle dispersion of the same absorbance of 1.0 at $395 \mathrm{~nm}$ for samples of different mean sizes, the number of absorbed photons by each CdS nanoparticle was higher when the mean size of the nanoparticles was smaller. An increase in the number of absorbed photons per CdS nanoparticle is known to cause the Auger recombination, ${ }^{21,22}$ leading to the nonlinear relationship. To minimize the effect of multiphoton absorption in the discussions, we fixed the excitation intensity in the experiments constant at $3.5 \mu \mathrm{J}$, at which the linear correlation between $\Delta \mathrm{Abs}$ at $t=0$ and excitation intensity for the largest CdS nanoparticles $(W=8)$ held and an efficient signal/noise ratio was obtained for the smallest $\mathrm{CdS}$ nanoparticles $(W=4)$.

III. Effect of Electron/Hole Quencher. To analyze the ultrafast dynamics in CdS nanoparticles in detail, it is essential to clarify whether the transient IR absorption is due to photoexcited electrons or holes. This can be achieved by examining the effect of electron or hole acceptors in the solutions on the relaxation behaviors. We used benzoquinone (BQ) and $p$-aminothiophenol (ATP) as an electron acceptor (electron quencher) and as a hole acceptor (hole quencher), respectively, as has already reported by Burda et al. ${ }^{8}$

Figure 4a shows transient IR absorption behaviors of dispersed $\mathrm{CdS}$ nanoparticles $(W=8)$ in $n$-heptane containing various concentrations of BQ with normalized decay curves in the inset. All of the decay curves can be fitted biexponentially. The addition of BQ clearly led to reduction of both amplitudes and lifetimes of the fast and slow relaxation components in the IR transient absorption. Figure $4 \mathrm{~b}$ shows BQ concentration, [BQ], dependence of the ratio of the lifetime of the faster decay component $\tau$ to $\tau_{0}$, where $\tau_{0}$ is the value of $\tau$ in the BQ free $\mathrm{CdS}$ nanoparticle dispersion. A linear relationship between $\tau_{0} / \tau$ and $[\mathrm{BQ}]$ corresponding to the Stern-Volmer equation, $1 / \tau=$ $1 / \tau_{0}+k_{\mathrm{q}}[\mathrm{BQ}]$, where $k_{\mathrm{q}}$ is the quenching constant, was obtained. This result suggests that the IR absorption reflects the electron concentration. From linear fitting in Figure $4 \mathrm{~b}$, the electron transfer (ET) rate constant, $k_{\mathrm{ET}}=k_{\mathrm{q}}[\mathrm{BQ}]$, was determined to be $4.3 \times 10^{11} \mathrm{~s}^{-1}$, which is ten times smaller than that reported for the ET rate between CdSe nanoparticles and BQ in toluene. ${ }^{8}$ The ET rate from CdS nanoparticles to methyl viologen $\left(\mathrm{MV}^{2+}\right)$ in water was reported to be on the order of $10^{12} \mathrm{~s}^{-1} \cdot{ }^{13}$ The small ET rate of the present system may be due to the existence of a water layer between $\mathrm{CdS}$ nanoparticles and AOT because direct adsorption of BQ on the CdS surface should be inhibited by the water layer.

Figure 5 shows the transient IR absorption behaviors of dispersed CdS nanoparticles $(W=8)$ in $n$-heptane containing various concentrations of ATP. As has been reported by Burda 

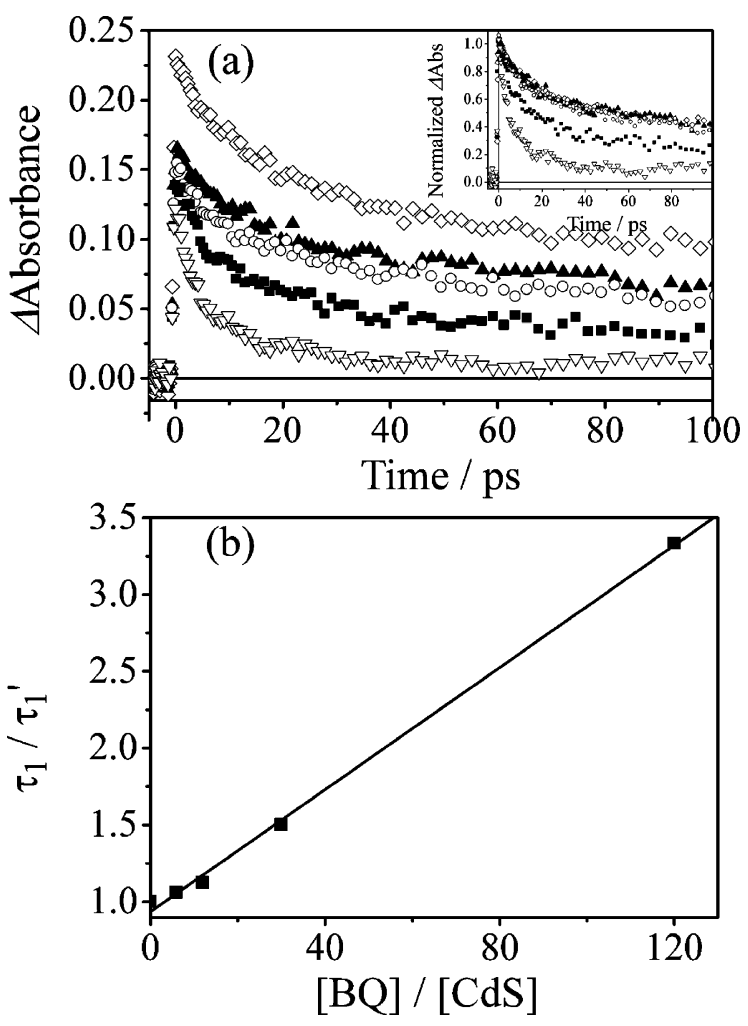

Figure 4. (a) $\mathrm{BQ}$ concentration dependence of photoinduced $\triangle \mathrm{Abs}$ relaxation dynamics of CdS nanoparticles $(W=8)$. Time-resolved IR transients correspond to the concentration ratios of $[\mathrm{BQ}]:[\mathrm{CdS}$ nanoparticle] $=0: 1$ (open diamonds), 6:1 (solid triangles), 12:1 (open circles), 30:1 (solid squares), and 120:1 (inverse open triangles). Inset: Normalized relaxation dynamics of $\Delta$ Abs. (b) Stern-Volmer plot for the lifetime of the fast component obtained from part a.

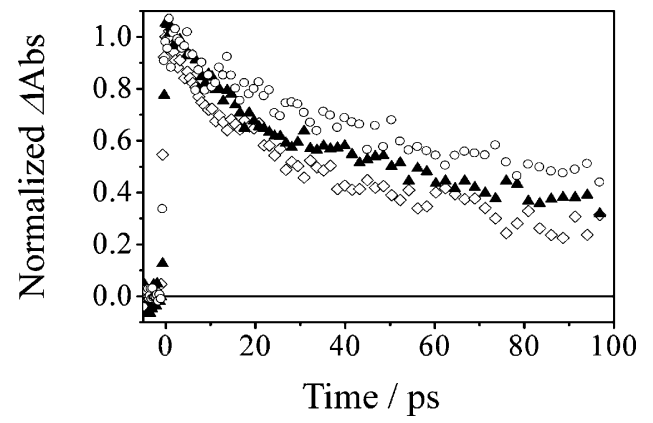

Figure 5. ATP concentration dependence of normalized photoinduced $\Delta$ Abs relaxation dynamics of CdS nanoparticles $(W=8)$. Time-resolved IR transients correspond to the concentration ratios of $[\mathrm{ATP}]:[\mathrm{CdS}$ nanoparticle $]=0: 1$ (open diamonds), 10:1 (solid triangles), and 100:1 (open circles).

et al., ${ }^{8}$ the lifetime of transient IR absorption of CdS nanoparticles became slightly longer when ATP was added. This can be explained by the reduced electron-hole recombination as a result of the removal of holes by ATP, a hole acceptor. However, it is also possible that ATP molecules are adsorbed on the surfaces of CdS nanoparticles so that the density of the electron traps at the surface was reduced. Actually, we also observed that the addition of thiophenol (TP), which is not a hole quencher but is adsorbed on CdS nanoparticles, also reduced the decay rate of transient absorption. Although we cannot determine at this stage which is the main factor responsible for the increase in the relaxation lifetime of transient IR absorption when ATP was added, it is clear that the IR absorbance is not directly related to the concentration of holes.
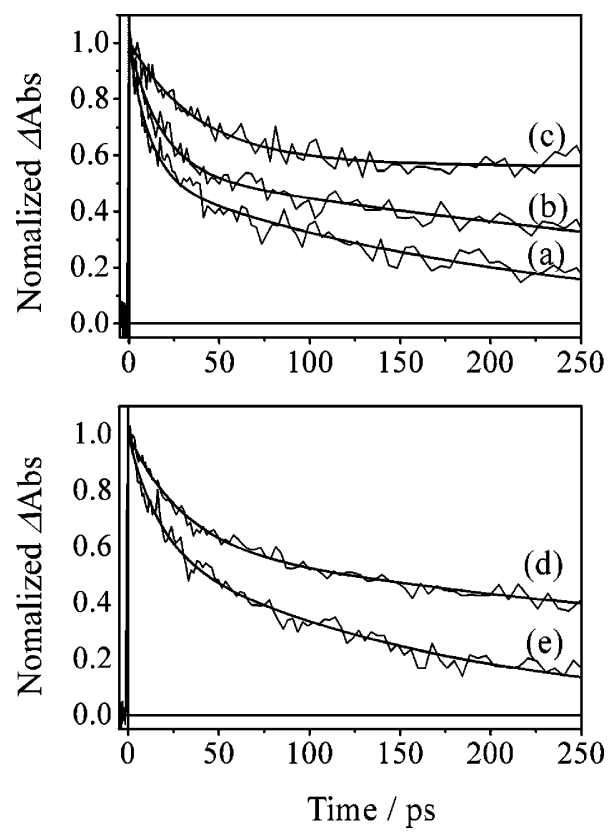

Figure 6. Size dependence of normalized femtosecond photoinduced $\triangle$ Abs relaxation dynamics of CdS nanoparticles: $W=$ (a) 4 , (b) 5 , (c) 6 , (d) 7, and (e) 8 . The results of the biexponential fitting are shown as solid curves.

From the above results, we can conclude that the mid-IR transient absorbance observed in the present system is due to the intraband excitation of photoexcited conduction electrons. Guyot-Sionnest et al. also reported intraband electron transitions in the IR range from 2.5 to $5 \mu \mathrm{m}$ for CdSe nanoparticle dispersions. ${ }^{2,3}$ Thus, the relaxation dynamics monitored by the present experiments are correlated to the dynamics of photogenerated free electrons.

IV. Size Dependence. The size dependence of the transient IR absorption behaviors of CdS nanoparticles was examined. Figure 6 shows the normalized transient IR absorption decays of CdS nanoparticles dispersed in $n$-heptane with mean diameter from $2.9(W=4)$ to $4.1 \mathrm{~nm}(W=8)$. The IR transient decays showed complicated relationships with the size of CdS nanoparticles. The lifetimes of fast and slow relaxation components for each IR absorption transient are summarized in Figure 7 and Table 2. In Figure 7b, the lifetimes of the slow relaxation component for the two samples with mean diameters of 3.4 $(W=6)$ and $3.5 \mathrm{~nm}(W=6.5)$ are not shown since the fitted lifetime values were more than $1 \mathrm{~ns}$, which exceeded the time window of the present system. The lifetimes of both the fast relaxation component, $\tau_{1}$, and the slow relaxation component, $\tau_{2}$, show "volcano"-type dependencies on the mean diameter of CdS nanoparticles. The lifetimes increased with an increase in size up to $3.4-3.5 \mathrm{~nm}$ and then decreased. The contribution from the fast decay became larger with a decrease in mean diameter, as shown in Table 2. The decrease in the contribution of the fast component as well as the increase in the lifetimes with an increase in the size of $\mathrm{CdS}$ nanoparticles can be explained by considering the decrease in the surface states, which act as recombination centers, because larger CdS nanoparticles have a smaller surface/volume ratio. A further increase in size led to a decrease in both the fast and slow lifetimes. This should be due to the increase in interior trap states such as crystal defects, which reduce the lifetime, with an increase in the size of CdS nanoparticles. In this scenario, the lifetime of photoexcited free electrons in $\mathrm{CdS}$ nanoparticles is determined by the balance between the densities of surface and interior trap 

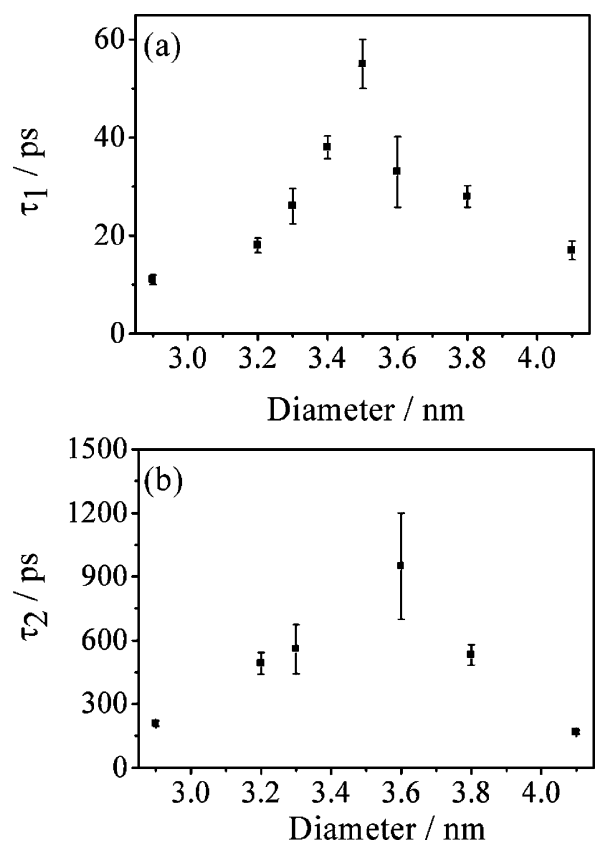

Figure 7. Size dependence of lifetimes of (a) fast and (b) slow relaxation components. The lifetimes of slower components for several CdS nanoparticles $(3.4 \mathrm{~nm}<$ mean size $<3.6 \mathrm{~nm})$ are not shown in part $\mathrm{b}$ since the values are above $1 \mathrm{~ns}$.

TABLE 2: Correlation between the Mean Diameter of CdS Nanoparticles and the Relaxation Lifetime for Fast and Slow Components

\begin{tabular}{cccrc}
\hline diameter/nm & $\tau_{1} / \mathrm{ps}$ & $A_{1} /\left(A_{1}+A_{2}\right)$ & $\tau_{2} / \mathrm{ps}$ & $A_{2} /\left(A_{1}+A_{2}\right)$ \\
\hline 2.9 & 11 & 0.47 & 207 & 0.53 \\
2.8 & 18 & 0.45 & 492 & 0.55 \\
3.3 & 26 & 0.43 & 559 & 0.57 \\
3.4 & 38 & 0.43 & $>1000$ & 0.57 \\
3.5 & 55 & 0.37 & $>1000$ & 0.63 \\
3.6 & 33 & 0.36 & 950 & 0.64 \\
3.8 & 28 & 0.38 & 532 & 0.62 \\
4.1 & 17 & 0.39 & 166 & 0.61
\end{tabular}

states in the CdS nanoparticles, and the sum of the contributions of surface and interior states seemed to be minimum at the size of ca. $3.5 \mathrm{~nm}$.

Although size dependencies of ultrafast photoexcited carrier dynamics have been investigated for several systems, including $\mathrm{CdS},{ }^{23} \mathrm{CdSe},{ }^{2,3} \mathrm{InP},{ }^{24}$ and $\mathrm{Cu}_{x} \mathrm{~S}^{25}$ nanoparticles and $\mathrm{CdS}$ nanorods, ${ }^{12}$ a "volcano"-type relationship between the electron relaxation lifetime and the size of the nanoparticles was observed for the first time in the present system. Such a unique behavior can be attributed to the unique shell structure around the CdS nanoparticles, i.e., $\mathrm{CdS}$ was covered by a highly dielectric water layer, a AOT reversed micelle layer, and a nonpolar heptane solvent. In addition, since the surface of the CdS nanoparticle was not stabilized by surface capping molecules, the effect of surface trap states became more obvious for the smaller CdS nanoparticles.

V. Effect of Surface Stabilization. To clarify the effect of surface stabilization on the ultrafast dynamics, the size dependence of free electron dynamics was investigated also for the thiol-capped CdS nanoparticles dispersed in $\mathrm{D}_{2} \mathrm{O}$. UV-visible absorption spectra of $\mathrm{CdS}$ nanoparticles before and after the thiol-termination treatment seem to be identical, indicating that the size of CdS nanoparticles was not changed during thiolcapping treatment and, then, neither condensation nor dissolution took place. To avoid strong IR absorption around $4 \mu \mathrm{m}$, two

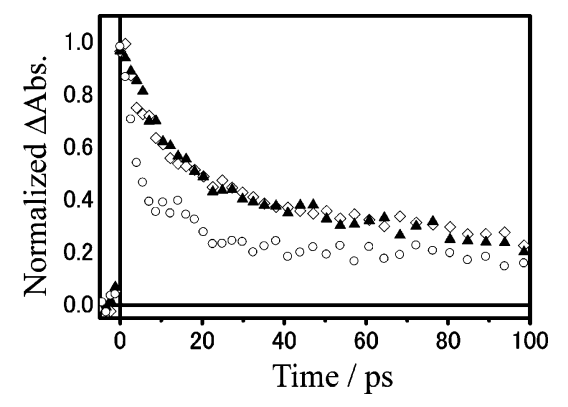

Figure 8. Photoinduced $\triangle \mathrm{Abs}$ relaxation dynamics of thiol-capped $\mathrm{CdS}$ nanoparticles $(4.0 \mathrm{~nm})$ dispersed in $\mathrm{D}_{2} \mathrm{O}$ (open diamonds), $\mathrm{D}_{2} \mathrm{O}$ containing $\mathrm{MV}^{2+}$ (open circles), and $\mathrm{D}_{2} \mathrm{O}$ containing TEOA (solid triangles). The probe wavelength is $3.2 \mu \mathrm{m}$.

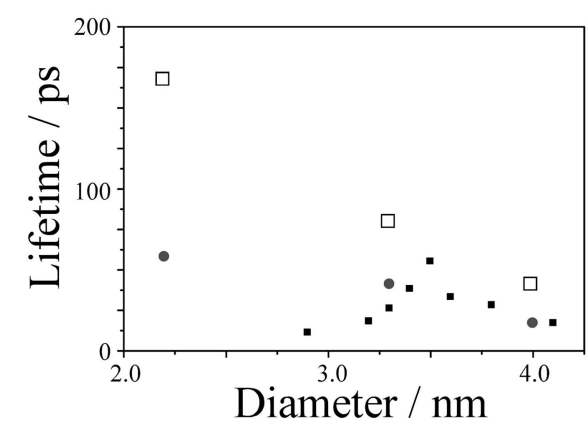

Figure 9. Size dependences of lifetime of fast relaxation components for thiol-capped CdS nanoparticles measured at two different probe wavelengths (open squares, $3.2 \mu \mathrm{m}$ probe; filled circles, $2.8 \mu \mathrm{m}$ probe); that for CdS nanoparticles in AOT reversed micelles (filled squares, $4.0 \mu \mathrm{m}$ probe) is also shown as a reference.

different probe wavelengths of $3.2(0.39 \mathrm{eV})$ and $2.8 \mu \mathrm{m}(0.44$ $\mathrm{eV})$ were used. Figure 8 shows the IR absorption decay behaviors of thiol-terminated $\mathrm{CdS}$ nanoparticles $(4.0 \mathrm{~nm})$ probed at $3.2 \mu \mathrm{m}$ in $\mathrm{D}_{2} \mathrm{O}$ with and without electron $\left(\mathrm{MV}^{2+}\right)$ or hole (triethanolamine: TEOA) acceptors. While the addition of an electron acceptor made the relaxation decay faster, the addition of a hole acceptor did not affect the relaxation behavior. The same results were also obtained when the probe wavelength was $2.8 \mu \mathrm{m}$, although the relaxation lifetimes depended on the probe wavelength. These results are essentially the same as those shown in Figures 4 and 5. Thus, the relaxation dynamics monitored at the probe wavelengths of 3.2 and $2.8 \mu \mathrm{m}$ was confirmed to be related to the dynamics of photogenerated free electrons.

Figure 9 shows size dependencies of the lifetimes of fast relaxation components of thiol-capped $\mathrm{CdS}$ nanoparticles measured at the two probe wavelengths stated above and uncapped $\mathrm{CdS}$ nanoparticles in AOT reversed micelles measured at 4.0 $\mu \mathrm{m}$ for comparison. The lifetimes of the slow relaxation component for thiol-capped CdS nanoparticles were too long ( $>1 \mathrm{~ns}$ ) to determine by the present experimental system, of which the maximum time delay is $1 \mathrm{~ns}$. In the case of the thiolcapped CdS nanoparticles, a "volcano"-type relationship between the relaxation lifetime and the size of CdS nanoparticles was no longer observed and the lifetime of the fast component monotonically increased as the size became smaller. Although the probe wavelengths were different for the time-resolved transient IR measurements at uncapped and thiol-capped CdS nanoparticles, the relationship between size and relaxation lifetime by thiol-termination was similar and the trend may be independent of the wavelength of the probe beam. Thus, we can conclude that the lifetime of photoexcited free electrons 
was increased by thiol-capping and this effect became more apparent when the size became smaller.

The modification of the surfaces of $\mathrm{CdS}$ nanoparticles by thiol molecules passivated the electron traps at surfaces of $\mathrm{CdS}$ nanoparticles. An increase in the relaxation lifetime of photoexcited electrons by passivation of surface electron traps with thiol-termination has also been reported for another system. ${ }^{26}$ However, several studies ${ }^{27-29}$ have indicated that thiol-cap termination leads to a decrease in the photoluminescence (PL) intensity for semiconductor nanoparticle systems, although the lifetime of photoexcited free electrons increases. ${ }^{26}$ Actually, We have also observed that the PL intensity decreased significantly by thiol-capping. ${ }^{30}$ Recently, Wuister et al. investigated the effect of thiol capping on the exciton luminescence of CdTe and CdSe nanoparticles. ${ }^{31}$ They found that the thiol-capping treatments resulted in an increase in luminescence for CdTe nanoparticles but quenching of CdSe nanoparticles. They concluded that the thiol adsorption resulted in the generation of a new hole trap channel and because of the lower energetic position of the valence band for CdSe than that of CdTe, trapping of the photogenerated holes took place more effectively at CdSe. Since the position of the valence band of $\mathrm{CdS}$ nanoparticles is expected to be even lower than that of $\mathrm{CdSe}$, the hole trapping to thiol is energetically more favorable at CdS. From the above discussions, it can be concluded that thiolcapping treatment not only deactivates surface electron trap states, resulting in longer lifetimes of free electrons, but also introduces new hole trap states, resulting in luminescence quenching, on the surfaces of CdS nanoparticles.

\section{Conclusion}

In summary, we have observed a "volcano"-type relationship between the size and the photoexcited free electron lifetime for "uncapped" CdS nanoparticle dispersions and shown that the balance between densities of surface and interior trap states was the origin of this relationship. A quite different size dependence of the photoexcited free electron lifetime was observed when thiol(thiolate)-cap molecules were introduced. The photoexcited free electron lifetime monotonically increased as the size decreased, indicating that the surface trap state was deactivated by the thiol-cap, although PL was efficiency decreased by the same treatments.

Acknowledgment. We thank Prof. Torimoto for helpful comments for the preparation of thiol-modified CdS nanoparticles and the dynamic laser scattering (DLS) measurements. This work was partially supported by Grants-in-Aid for Scientific Research on Priority Areas (432) from the Ministry of Education, Culture, Sports, Science and Technology (MEXT), Japan. This work was also supported by PRESTO of JST (Japan Science and Technology Corporation).
Supporting Information Available: Structures of CdS nanoparticles prepared by reversed micelle method are speculated from results of FT-IR measurements. This material is available free of charge via the Internet at http://pubs.acs.org.

\section{References and Notes}

(1) Eychmuller, A. J. Phys. Chem. B 2000, 104, 6514.

(2) Guyot-Sionnest, P.; Hines, M. A. Appl. Phys. Lett. 1998, 72, 686.

(3) Guyot-Sionnest, P.; Shim, M.; Matranga, C.; Hines, M. Phys. Rev. B 1999, 60, R2181.

(4) Klimov, V. I.; Schwarz, C. J.; McBranch, D. W.; Leatherdale, C. A.; Bawendi, M. G. Phys. Chem. B 1999, 60, R2177.

(5) Klimov, V. I. J. Phys. Chem. B 2000, 104, 6112.

(6) Klimov, V. I.; Mikhailovsky, A. A.; McBranch, D. W.; Leatherdale, C. A.; Bawendi, M. G. Phys. Chem. B 2000, 61, R13349.

(7) Xu, S.; Mikhailovsky, A. A.; Hollingsworth, J. A.; Klimov, V. I. Phys. Chem. B 2002, 65, 045319/1.

(8) Burda, C.; Link, S.; Mohamed, M.; El-Sayed, M. J. Phys. Chem. B 2001, 105, 12286.

(9) Burda, C.; Green, T. C.; Link, S.; El-Sayed, M. A. J. Phys. Chem. B 1999, 103, 1783 .

(10) Htoon, H.; Hollingsworth, J. A.; Dickerson, R.; Klimov, V. I. Phys. Rev. Lett. 2003, 91, 227401/1.

(11) Htoon, H.; Hollingworth, J. A.; Malko, A. V.; Dickerson, R.; Klimov, V. I. Appl. Phys. Lett. 2003, 82, 4776.

(12) Yu, P.; Nedeljkovic, J. M.; Ahrenkiel, P. A.; Ellingson, R. J.; Nozik, A. J. Nano Lett. 2004, 4, 1089.

(13) Logunov, S.; Green, T.; Marguet, S.; El-Sayed, M. A. J. Phys. Chem. A 1998, 102, 5652.

(14) Zhang, J. Z. Acc. Chem. Res. 1997, 30, 423.

(15) Zhang, J. Z. J. Phys. Chem. B 2000, 104, 7239

(16) Nakanishi, T.; Ohtani, B.; Uosaki, K. Jpn. J. Appl. Phys. 1997, 36, 4053 .

(17) Torimoto, T.; Maeda, K.; Maenaka, J.; Yoneyama, H. J. Phys. Chem. 1994, 98, 13658.

(18) Ebina, K.; Yagi, I.; Noguchi, H.; Uosaki, K. Chem. Lett. 2004, 33, 604.

(19) Uosaki, K.; Okamura, M.; Ebina, K. Faraday Discuss. 2004, 125, 39.

(20) Steigerwald, M. L.; Alivisatos, A. P.; Gibson, J. M.; Harris, T. D.; Kortan, R.; Muller, A. J.; Thayer, A. M.; Duncan, T. M.; Douglass, C. D.; Brus, L. E. J. Am. Chem. Soc. 1988, 110, 3046.

(21) Klimov, V. I.; McBranch, D. W. Phys. Chem. B 1997, 55, 13173.

(22) Burda, C.; Link, S.; Mohamed, M. B.; El-Sayed, M. J. Chem. Phys. 2002, 116, 3828 .

(23) Roberti, T. W.; Cherepy, N. J.; Zhang, J. Z. J. Chem. Phys. 1998 $108,2143$.

(24) Blackburn, J. L.; Ellingson, R. J.; Micic, O. I.; Nozik, A. J. J. Phys. Chem. B 2003, 107, 102.

(25) Lou, Y.; Samia, A. C. S.; Cowen, J.; Banger, K.; Chen, X.; Lee, H.; Burda, C. Phys. Chem. Chem. Phys. 2003, 5, 1091.

(26) Jeong, S.; Achermann, M.; Nanda, J.; Ivanov, S.; Klimov, V. I.; Hollingsworth, J. A. J. Am. Chem. Soc. 2005, 127, 10126.

(27) Uyeda, H. T.; Medintz, I. L.; Jaiswal, J. K.; Simon, S. M.; Mattoussi, H. J. Am. Chem. Soc. 2005, 127, 3870.

(28) Herron, N.; Wang, Y.; Eckert, H. J. Am. Chem. Soc. 1990, 112, 1322.

(29) Gao, X.; Chan, W. C. W.; Nie, S. J. Biomed. Opt. 2002, 7, 532.

(30) Okamura, M.; Ebina, K.; Akimoto, S.; Yamazaki, I.; Uosaki, K. J. Photochem. Photobiol. A 2006.

(31) Wuister, S. F.; Donega, C. d. M.; Meijerink, A. J. Phys. Chem. B 2004, 108, 17393. 